\title{
Cystic Lymphangioma of the Adrenal Gland: A Case Report and a Review of the Literature
}

\author{
Faten hadjkacem', Mouna Ammar ${ }^{1}$, Ayman Maalej', Mouna Elleuch ${ }^{1, *}$, Nessrine Cheikrouhou ${ }^{1}$, Mouna Mnif ${ }^{1}$, \\ Nabila Rekike ${ }^{1}$, Fatma Mnif ${ }^{1}$, Nadia Charfi $^{1}$, Mohamed Abid ${ }^{1}$ \\ ${ }^{1}$ Department of endocrinology, Hedi Chaker Hospital, Sfax, Tunisia \\ ${ }^{2}$ Department of radiology, Habib Bourguiba Hospital, Sfax, Tunisia \\ *Corresponding author: elleuch_mouna@yahoo.fr
}

Received February 05, 2015; Revised March 11, 2015; Accepted March 27, 2015

\begin{abstract}
Adrenal lymphangiomas, also known as cystic adrenal lymphangiomas are benign vascular lesions, most often found incidentally during abdominal imaging studies, abdominal surgery or at autopsy. We present a case of a 45-year-old female who was evaluated for recurrent abdominal pain. Imaging studies revealed a large cystic lesion in the right adrenal region considered as a hydatid cyst. Right adrenalectomy was performed. Histopathologic examination demonstrates that the definite diagnosis is cystic lymphangioma of the right adrenal gland.
\end{abstract}

Keywords: adrenal gland, lymphangioma, adrenal cyst, hydatid cyst

Cite This Article: Faten Hadjkacem, Mouna Ammar, Ayman Maalej, Mouna Elleuch, Nessrine Cheikrouhou, Mouna Mnif, Nabila Rekike, Fatma Mnif, Nadia Charfi, and Mohamed Abid, "Cystic Lymphangioma of the Adrenal Gland: A Case Report and a Review of the Literature.” American Journal of Medicine Studies, vol. 3, no. 1 (2015): 1-3. doi: 10.12691/ajms-3-1-1.

\section{Introduction}

Adrenal cystic lesions are uncommon with an incidence of approximately $0.06 \%$ in the general population. Adrenal cysts are classified as endothelial cysts, pseudocysts, epithelial cysts, and parasitic cysts. Endothelial cysts ( $20 \%$ to $32 \%$ of all adrenal cysts) are subdivided into two subgroups: lymphangiomatous and angiomatous cysts. Adrenal cystic lymphangioma (ACL) is a very rare benign vascular lesion. Most of the ACLs reported are asymptomatic and often incidentally identified during radiological investigation or surgery for unrelated causes, or at autopsy. Occasionally, symptoms can occur in response to ACLs and they are typically related to the overall mass of the cyst and include a palpable mass, gastrointestinal discomfort and abdominal pain.

Although current advancements in imaging techniques have provided the possibility of clinical characterization of adrenal cystic lesions, a range of adrenal neoplasms remained in their clinical differential diagnosis, and thus necessitating surgical resection.

In this report, we describe an ACL considered as a hydatid cyst because of its radiological appearance, with an emphasis on the symptoms, differential diagnosis, and treatment.

\section{Case Report}

A 45-year-old woman presented with recurrent abdominal pain since 3 months. She reported a past medical history of systemic hypertensive disease since 2 years. Clinical examination was normal with stabile hypertension.

Abdominal ultrasonography (US) showed a multicystic mass in the right adrenal region. The CT scan revealed an hypo-dense, a multicystic mass measuring $6 \times 5 \mathrm{~cm}$ in the right adrenal bed, with slightly mural enhancing after administered with contrast medium and calcifications of the cystic wall. The pictures obtained were very evocative of hydatid cyst (Figure 1).

Hydatid serology was negative.

An adrenal evaluation was performed and was within normal limits. These results excluded a functional cystic tumor.

On the basis of these findings, surgical excision was carried out with a subcostal flank incision, grossly showing an $8 \times 7 \mathrm{~cm}$ well-defined, multiloculated cystic lesion. The cyst was excised and a right adrenalectomy was perfomed without any intraoperative complications.

Histologically, the cyst showed a multicystic architecture with dilated spaces lined with flattened, simple endothelial cells. The cystic spaces often contained calcifications.

There were no hydatid membranes and no evidence of malignance was identified in the adrenal mass. The histopathological diagnosis was a cystic lymphangioma in the right adrenal gland.

The patient's postoperative course was uneventful. Three months after the operation, she did not have abdominal pain and she experienced no signs or symptoms of endocrine dysfunction. 

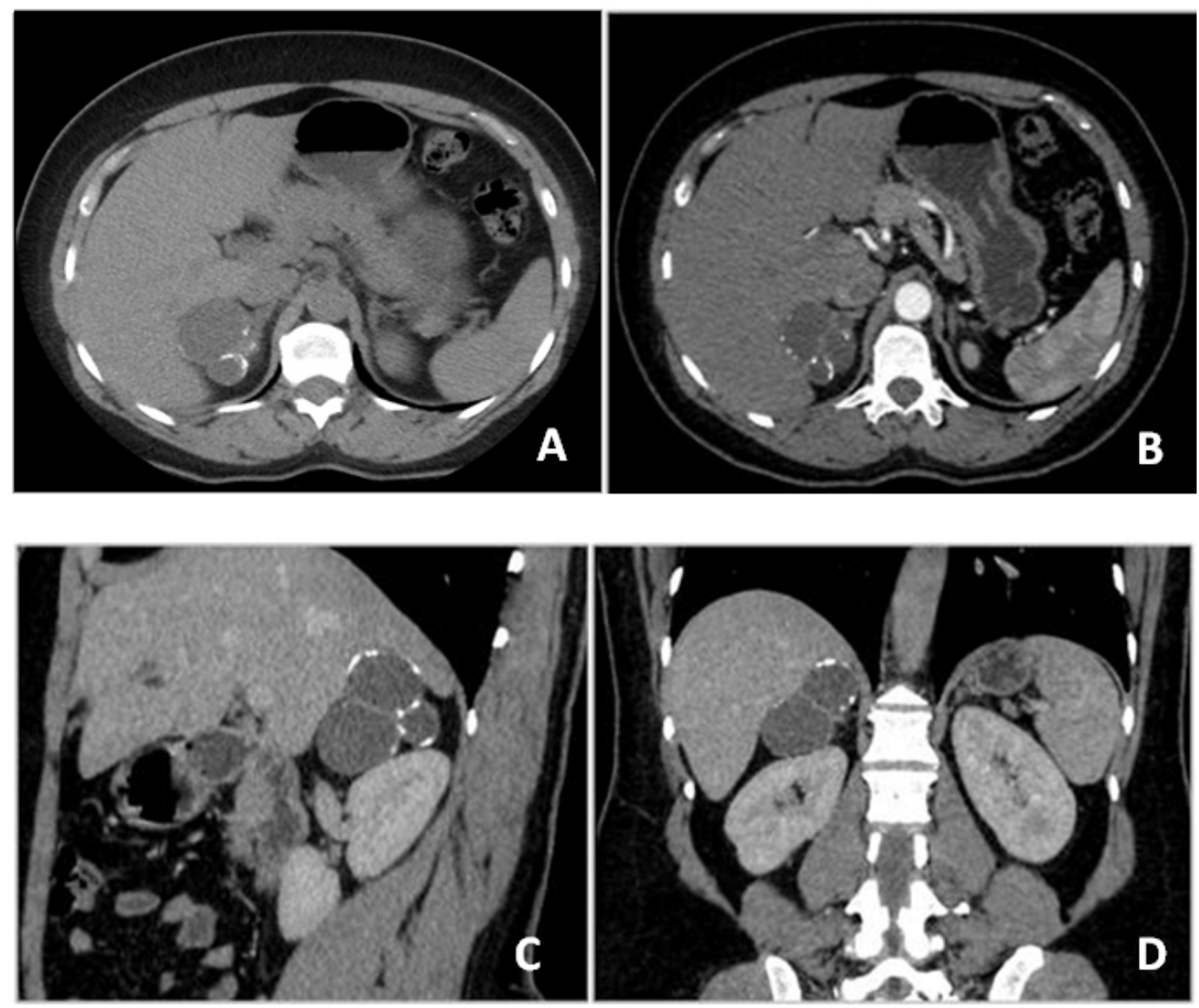

Figure 1. (A) Computed tomography scan shows a large hypo-dense multicystic lesion measuring $5 * 6 \mathrm{~cm}$ with calcifications in the cystic wall; axial section. (B, C, D) Post-contrast CT scan demonstrates a non-enhancing lesion; axial, sagittal and coronal sections

\section{Discussion}

ACL is a benign vascular lesion and a subtype of endothelial adrenal cysts. Cystic lymphangioma developing in the adrenal gland is extremely rare. The first case of ACL had been reported in 1965, recently less than 50 immunohistochemically proven cases have been reported in the literature. The largest series, reported in 2011 by Ellis et al. includes 9 immunohistochemically proven ACLs [1].

Lymphangioma is primarily located in the neck or axilla (95\%), the other $5 \%$ of cases are discovered in the abdominal cavity, including the mesentery of the small intestine, the omentum, mesocolon and retroperitoneum. Lymphangioma can occur at all ages, with the peak incidence between the third and sixth decade of life as seen in our case [2].

In addition, the ACL in our case affected female patient and arise in the right-sided adrenal as seen in Ellis et al's observations that ACL had a significant female predisposition and twice more frequently involved rightsided adrenal [1].

The pathogenesis of ACL is still controversial. The most favored theories are that these lesions stem from continued growth of ectopic or malformed lymphatic tissues or represent hyperplastic reaction to inflammation or a lymphatic hamartoma. Blockage of draining lymphatics, proximal dilatation of the lymphatic system, and even traumas have also been suggested as causes of ACL [1].

ACLs are macroscopically well-defined multilocular, thin-walled cystic lesions and are filled with non viscous, clear, yellow-brown colored fluid. Histologically, they are multicystic lesions formed by irregular dilated spaces lined with flattened endothelial cells. The cystic spaces are empty or filled with acellular, homogeneous proteinaceous material with occasional calcification [3]. The endothelial cells lining these cavities are immune-reactive for vascular cell markers such as CD31 and CD34. In addition, the D240 monoclonal antibody to the transmembrane mycoprotein only recognizes the lymphatic endothelium, thus suggesting that it is a specific marker for cells of lymphatic origin. Immunostains are usually not needed for the diagnosis of ACL, but co expression of D2-40, CD31, CD34, and factor VIII-related antigen by the lining cell can be used to confirm its lymphatic origination [4].

ACLs are usually asymptomatic. However, symptoms can occasionally occur and they are typically related to the overall mass of the cyst. The most common symptoms are abdominal or flank pain, gastrointestinal discomfort, palpable mass and arterial hypertension and palpitation especially in functional cysts $[5,6]$.

On ultrasound (US), ACL is a well-marginated, anechoic lesion typically located on the suprarenal area and which can show shadows and internal echoes, when calcifications or internal debris are present [1-7]. On CT scan the mass is identified as a hypodense, non-enhanced 
lesion with smooth borders and thin wall ( $<3,5 \mathrm{~mm})$. US and CT scans can reveal peripheral calcifications of the cystic wall in $15 \%$ of cases. Such calcifications in the cystic wall were observed in our case.

On Magnetic resonance imaging (MRI), ACLs are typically homogeneous, with low signal intensity at T1weighted images and high signal intensity at T2-weighted images without internal enhancement. MRI is more sensitive for detecting intracystic hemorrhage and complicated cysts which are shown by high signal intensity on both T1 and T2 weighted images [8].

Aspiration of the contents of adrenal cysts is recently recommended for their diagnosis and management, if it brought a fluid without any malignant cells but lymphoid cells and milk-like lymph are present, the diagnosis of ACL can be made [9].

The differential diagnosis of ACL includes cystic benign or malignant adrenocortical neoplasms as well as other cyst types. Pseudocysts represent the most common type of adrenal cyst and have been thought to be a result of hemorrhage within a tumor or hemorrhage into adrenal parenchyma due to trauma, toxic or infectious process. [1] Hydatid cyst of the adrenal gland is exceptional and accounts for only $6 \%$ to $7 \%$ of all adrenal cysts but it should be considered in endemic areas [10].

Current treatment recommendations for ACLs are that small asymptomatic nonfunctioning lesions can simply be observed, whereas large cysts (5 cm or more in diameter), cyst with high density on unenhanced CT $(>10 \mathrm{HU})$ or delayed wash-out of the contrast medium, significant growth of the cyst on serial imaging, Functional cysts, symptomatic cysts and cysts which can cause complications such as hemorrhage, rupture, or infection should be excised [11].

In conclusion, ACL is a very rare entity. To date, approximately 50 cases of adrenal gland lymphangioma diagnosed by immunohistochemistry have been reported in the literature. Most of the ACLs reported are asymptomatic but symptoms can occasionally occur and they are typically related to the overall mass of the cyst. Imaging can characterize the cystic nature of these lesions. However, it sometimes fails to establish a specific diagnosis preoperatively. So definite diagnosis relies on histologic and immunohistochemical examination.

\section{Conflict of Interest}

None to declare.

\section{References}

[1] C. L. Ellis, P. Banerjee, E. Carney, R. Sharma, and G. J. Netto. Adrenal lymphangioma: clinicopathologic and immunohistochemical characteristics of a rare lesion. Human Pathology( 2011);( 42): 1013-1018.

[2] Khoda J, Hertzanu Y, Sebbag G, Lantsberg L, Barky Y. Adrenal cysts: diagnosis and therapeutic approach. Int Surg. (1993); (78): 239-242.

[3] J.M. Longo, S.Z. Jafri, andK. B. Bis, “Adrenal lymphangioma: a case report,”. Clinical Imaging.(2000); (24): 104106.

[4] Kalof AN, Cooper K. D2-40 immunohistochemistry-so far. Adv Anat Pathol. (2009); (16): 62-4.

[5] L. A. Erickson, R. V. Lloyd, R. Hartman, and G. Thompson. Cystic adrenal neoplasms. Cancer. (2004);(101): 1537-1544.

[6] Y. Tanuma, M. Kimura, and S. Sakai. Adrenal cyst: a review of the japanese literature and report of a case. International Journal of Urology. (2001); (8): 500-503.

[7] J.M. Longo, S.Z. Jafri, andK. B. Bis. Adrenal lymphangioma: acase report. Clinical Imaging. (2000);(24): 104-106.

[8] Y.-K. Guo, Z.-G. Yang, Y. Li et al.Uncommon adrenal masses: CT and MRI features with histopathologic correlation. European Journal of Radiology.(2007); (62): 359-370.

[9] Satou T, Uesugi T, Nakai Y, Hayashi Y, Imano M, Hashimoto S. Case of adrenal lymphangioma with atypical lymphocytes in aspirate cytology. Diagn Cytopathol. (2003); ( 29): 87-90.

[10] Goel MC, Agarwal MR, Misra A. Percutaneous drainage of renal hydatid cyst: early results and follow-up. Br J Urol. (1995); (75):724-8.

[11] A. A. Kasperlik-Załuska, M. Otto, A. Cichocki et al.1,161 patients with adrenal incidentalomas: indications for surgery. Langenbeck’s Archives of Surgery. (2008); (393): 121-126. 